\title{
Modelagem Hidrológica em uma Sub-bacia Hidrográfica do Baixo Rio Araguaia, TO
}

\section{Hydrological Modeling in a watershed of the Lower Araguaia River Basin, TO}

\author{
Marcelo Ribeiro Viola ${ }^{1 *}$, Carlos Rogério de Mello ${ }^{2}$, Marcos Giongo ${ }^{1}$, Samuel Beskow ${ }^{3}$ e \\ André Ferreira dos Santos ${ }^{1}$
}

${ }^{1}$ Engenharia Florestal, Universidade Federal do Tocantins, C.P. 66, 77.402-970, Gurupi, TO Brasil . ${ }^{2}$ Departamento de Engenharia, Universidade Federal de Lavras, C.P. 3037, 37.200-000, Lavras, MG Brasil . ${ }^{3}$ Centro de Desenvolvimento Tecnológico, Universidade Federal de Pelotas, 96.010-170, Pelotas, RS -Brasil.

\begin{abstract}
Hydrological simulation is an important tool for water resources management since it allows for practitioners to evaluate the impacts of anthropic activities and climatic changes on water availability. The Lontra River watershed is situated in the Lower Araguaia River Basin which is an important economic region of Northern Tocantins State. The understanding of its hydrological features is fundamental for the development of environmental studies for supporting the decision-making related to the water resources management as strong pressure has been observed due to both the agricultural frontier expansion and installed economic center. The LASH hydrological model (stands for Lavras Simulation of Hydrology) is characterized as a deterministic, semi conceptual and spatially distributed model and has been successfully applied in watersheds located in Southeastern Brazil. It was found in this study that the model was able to adequately capture the overall hydrological regime in the studied watershed. Three statistical coefficients used for measuring the model goodness-of-fit, Nash-Sutcliffe (CNS), Log (CNS) and determination coefficient $\left(R^{2}\right)$, have shown values greater than $0.74,0.80$ and 0.90, respectively. The simulated flow duration curve presented a good fit in relation to the observed one, with small errors for prediction of minimum and maximum stream flows. Thus, we can be conclude that LASH model simulated properly the hydrological regime in the Lontra River Watershed and it can be applied for either evaluation water availability or planning and management of water resources in the Lower Araguaia River Basin.
\end{abstract}

Key-words: Hydrology simulation, Lontra river basin, Water resources management

\section{INTRODUÇÃO}

$\mathrm{O}$ entendimento das inter-relações associadas aos fenômenos naturais que envolvem o ciclo hidrológico possibilitou o desenvolvimento de modelos matemáticos que descrevem a dinâmica da água nos ecossistemas naturais. Recentemente, o detalhamento das características físicas pela associação com técnicas de geoprocessamento e de sensoriamento remoto, levou ao aperfeiçoamento da modelagem dos sistemas hidrológicos. Neste contexto, os modelos hidrológicos de base conceitual e distribuída apresentam-se como uma importante ferramenta de análise ambiental, com possibilidade de aplicação para previsão de vazões, avaliação de cenários climáticos e de uso do solo, estudos sobre disponibilidade hídrica, entre outros. Este tipo de investigação científica é relevante no contexto hidrológico e tem sido abordado por diferentes modelos hidrológicos e em diferentes realidades físicas, podendo-se destacar: SWAT no Uruguai (Stackelberg et al., 2007), NRM3 no Quênia (Notter et al., 2007), AGNPS no sul da Itália (Licciardello et al., 2007), MGB no sul, sudeste e norte do Brasil (Collischonn et al., 2005; Nóbrega et al., 2011; Ribeiro Neto et al., 2008) e LASH no sudeste do Brasil (Mello et al., 2008;

Author for correspondence: mrviola@uft.edu.br 
Viola et al., 2009; Beskow et al., 2011). O Estado do Tocantins pertence à Região Norte do Brasil e está inserido na bacia hidrográfica do TocantinsAraguaia. Esta é a mais extensa área de drenagem totalmente inserida em território brasileiro, com $918.822 \mathrm{~km}^{2}$, e a segunda maior em potencial hidroenergético instalado, respondendo por $16 \%$ da matriz energética nacional (ANA, 2012). O regime hidrológico é identificado por um pronunciado período de vazante durante o qual a disponibilidade hídrica superficial é bastante reduzida e que se segue com uma estação de cheia, caracterizando a variabilidade de descargas dos sistemas fluviais tropicais (Aquino et al., 2009). De acordo com Latrubesse e Stevaux (2002) a bacia do alto rio Araguaia se estende desde a cabeceira até Registro do Araguaia, onde tem início a média bacia, prolongando-se até o município de Conceição do Araguaia, que marca o início da bacia do baixo rio Araguaia, após a planície do Bananal, cerca de $500 \mathrm{~km}$ a montante da confluência com o rio Tocantins.

Estão inseridos na bacia hidrográfica do rio Tocantins-Araguaia dois importantes biomas brasileiros, o Cerrado e a Amazônia. Em regiões de transição entre diferentes biomas podem ocorrer áreas de tensão ecológica, nas quais tipologias vegetais de regiões fitoecológicas distintas competem pelo mesmo ambiente fisiográfico (SEPLAN, 2011). Estas são importantes regiões ecológicas, sensíveis a mudanças climáticas (Malanson, 1997), de ampla biodiversidade e hábitat para diversas espécies (Henry, 2003), podendo inclusive apresentar características únicas e próprias (Kark e Rensburg, 2006). O local de estudo compreende a sub-bacia hidrográfica do rio Lontra, localizada na região Norte do Tocantins, na bacia do baixo rio Araguaia. Nesta região, ocorrem áreas de tensão ecológica na transição entre Cerrado, Floresta Estacional e Floresta Ombrófila, originando ambientes de alta taxa de endemismo e de ocorrência de espécies raras (SEPLAN, 2011). A hidrologia de bacias hidrográficas inseridas neste ambiente apresenta caráter peculiar, em vista da heterogeneidade de ambientes, e estudos a seu respeito são escassos. Percebe-se, portanto, que o entendimento e a capacidade de predição do regime hidrológico de sub-bacias hidrográficas inseridas no baixo rio Araguaia é altamente relevante no contexto da gestão de recursos hídricos. O modelo hidrológico Lavras Simulation of Hydrology (LASH) é conceituado como determinístico, semiconceitual e distribuído. Este modelo foi desenvolvido para a simulação hidrológica em bacias hidrográficas com limitação qualitativa de dados e tem sido aplicado com bons resultados em bacias hidrográficas da Região Sudeste do Brasil. Viola et al. (2009) aplicaram o modelo LASH para simular a hidrologia da bacia do rio Aiuruoca, de $2.095 \mathrm{~km}^{2}$, obtendo resultados de alta qualidade para as vazões máximas, mínimas e de referência. Mello et al. (2008) avaliaram a aplicabilidade do modelo LASH para simular os impactos hidrológicos frente a cenários de uso do solo na região de cabeceira do rio Grande, concluindo que o modelo apresentou boa acurácia na simulação do escoamento e que pode ser aplicado para a simulação dos impactos hidrológicos associados a modificações da cobertura vegetal. Beskow (2009) desenvolveu uma estrutura SIG para o modelo LASH e obteve bons resultados na simulação hidrológica da bacia hidrográfica do ribeirão Jaguara $\left(32 \mathrm{~km}^{2}\right)$. Viola (2011) acoplou o modelo LASH ao modelo climático regional Eta-CPTEC/HadCM3 para simular os possíveis impactos hidrológicos do cenário climático SRES A1B na hidrologia da região de cabeceira da bacia do rio Grande, no sul de Minas Gerais, tendo sido obtidos resultados importantes no tocante ao manejo dos recursos hídricos, tais como tendência ao prolongamento da vazante e maior concentração das chuvas em janeiro. Neste contexto, objetivou-se aplicar o modelo hidrológico LASH para a simulação hidrológica, com passo diário, na sub-bacia hidrográfica do rio Lontra, conceituada como importante região ecológica do norte do Tocantins e afluente direto pela margem direita do baixo rio Araguaia. Em termos específicos, após a calibração e a validação do modelo hidrológico, objetivou-se avaliar o erro de estimativa das principais vazões de referências obtidas da curva de permanência, e a sua performance na simulação contínua de vazões pelo coeficiente estatístico de Nash-Sutclife $\left(\mathrm{C}_{\mathrm{NS}}\right)$ e sua versão logarítmica $\left(\log \left(\mathrm{C}_{\mathrm{NS}}\right)\right)$, tendência média do estimador (D) e coeficiente de correlação $\left(\mathrm{R}^{2}\right)$.

\section{MATERIAL E MÉTODOS}

Localizada no norte do estado do Tocantins, a bacia hidrográfica do rio Lontra é uma das principais sub-bacias de drenagem pela margem direita do rio Araguaia. O clima predominante de acordo com a classificação climática de Thornthwaite é úmido com moderada deficiência 
hídrica no inverno (B1wA'a'), com evapotranspiração potencial entre 1.400 e 1.700 $\mathrm{mm}$, sendo de 390 a $480 \mathrm{~mm}$ nos três meses consecutivos de temperatura mais elevada (SEMADES, 2011). A temperatura média anual é de $25,0^{\circ} \mathrm{C}$, com a média da máxima e da mínima mensais situadas, respectivamente, entre $32,1^{\circ} \mathrm{C}$ e $20,1^{\circ} \mathrm{C}$. A lâmina média anual precipitada é da ordem de $1.852 \mathrm{~mm}$, com variação entre $1.357 \mathrm{~mm}$ (2007) e $2.893 \mathrm{~mm}$ (1985). A Figura 1 apresenta a marcha mensal da precipitação e temperaturas média, mínima e máxima, para a Estação Meteorológica de Araguaína, do Instituto Nacional de Meteorologia (INMET), entre 1985 e 2009 (BDMEP, 2012).

Para a finalidade deste estudo, a seção de controle estabelecida para a delimitação da bacia hidrográfica foi no posto fluviométrico da Companhia de Pesquisa em Recursos Minerais (CPRM) do município de Piraquê, localizada nas coordenadas de latitude $6^{\circ} 40^{\prime} 18^{\prime}$ 'sul e de longitude $48^{\circ} 28^{\prime} 11^{\prime \prime}$ oeste, totalizando área de drenagem de $3.470 \mathrm{~km}^{2}$. Nesta bacia hidrográfica está inserido um importante pólo econômico da região norte do Tocantins, abrangendo de forma total ou parcial os municípios de Aragominas, Araguaína, Araguanã, Babaçulândia, Carmolândia, Piraquê,
Wanderlândia e Xambioá. Na Figura 2 está apresentada a inserção da bacia hidrográfica do Tocantins-Araguaia no contexto brasileiro com destaque para a bacia hidrográfica do rio Lontra e a ocorrência dos biomas brasileiros, conforme IBGE (2004).

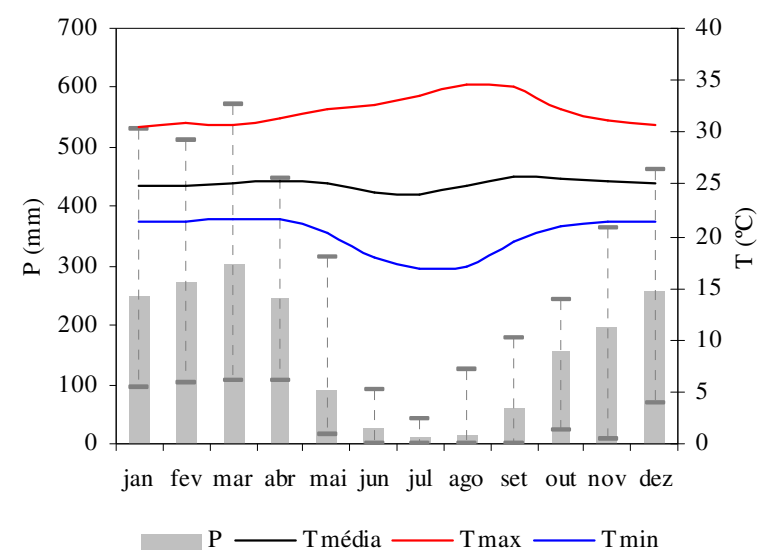

Figura 1 - Precipitação média mensal $(\mathrm{P})$ e respectiva faixa de variação, temperatura média mensal $\left(\mathrm{T}_{\text {média }}\right)$ e média das temperaturas máxima $\left(\mathrm{T}_{\max }\right)$ e mínima mensais $\left(\mathrm{T}_{\min }\right)$.
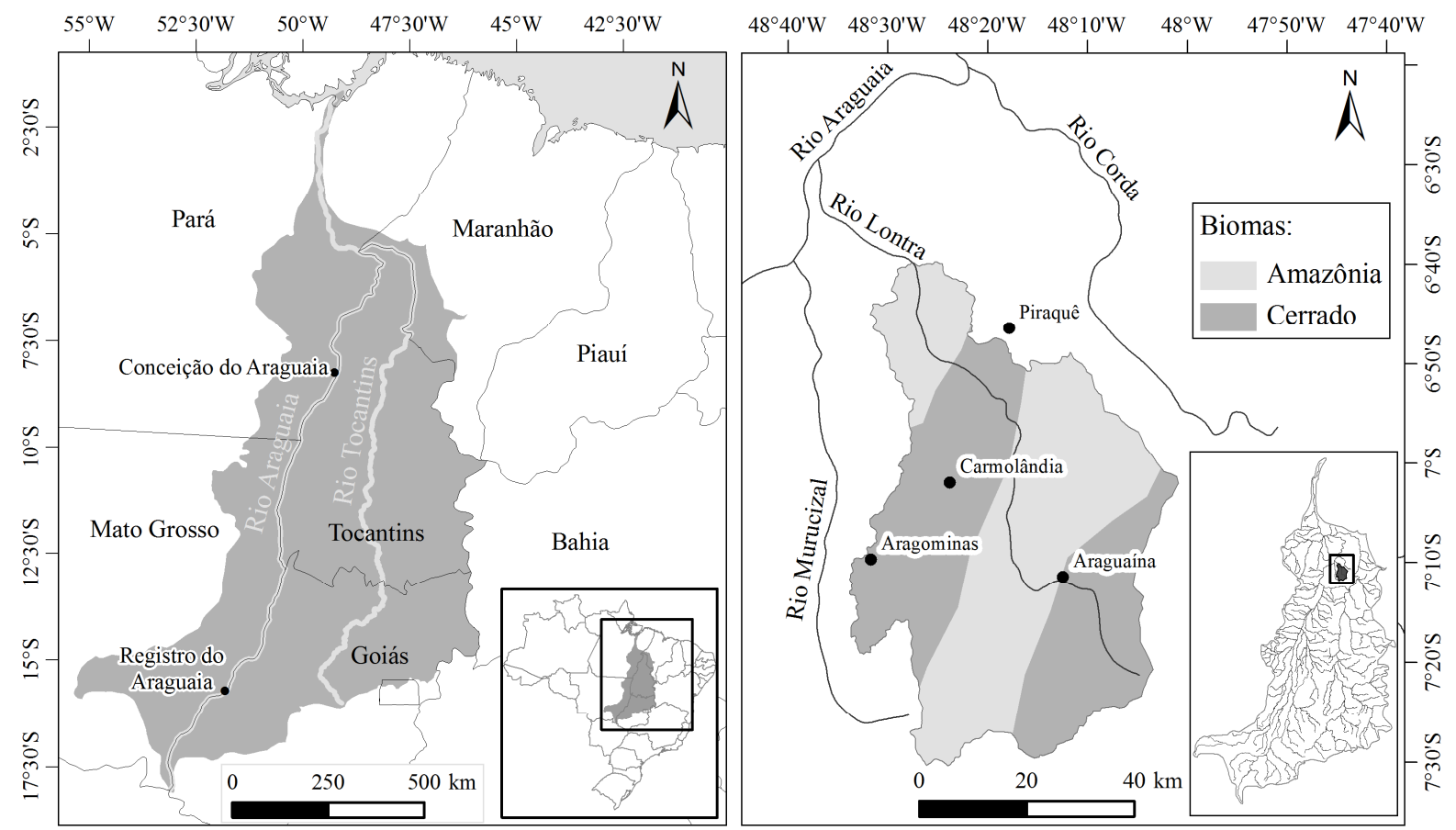

Figura 2 - Localização da bacia hidrográfica do rio Tocantins-Araguaia no território brasileiro com destaque para a bacia hidrográfica do rio Lontra, biomas, hidrografia e sedes municipais. 
Para aplicação do modelo hidrológico, estruturouse um banco de dados hidrometeorológico diário. Foram adquiridas, do Sistema de Informações Hidrológicas da Agência Nacional de Águas, séries históricas pluviométricas das estações de Piraquê (648002), Muricilândia (748003), Faz. Primavera (748002) e Wanderlândia (647001), além dos registros fluviométricos da estação de Piraquê (28240000) (HIDROWEB, 2012). Para alimentar o módulo de evapotranspiração do modelo LASH, que considera o método de Penman-Monteith, foram aplicados os elementos meteorológicos da estação do INMET localizada em Araguaína (BDMEP, 2012). Na Figura 2a está apresentada a localização dos postos de monitoramento hidrometeorológico aplicados a este estudo, hidrografia, sub-bacias para a modelagem distribuída e sedes municipais.

O uso do solo na bacia hidrográfica do rio Lontra é marcado pela atividade pecuária, que responde por $39 \%$ do uso consuntivo de água, basicamente atendido pelo manancial superficial. Ademais, prevalece a explotação de água subterrânea para

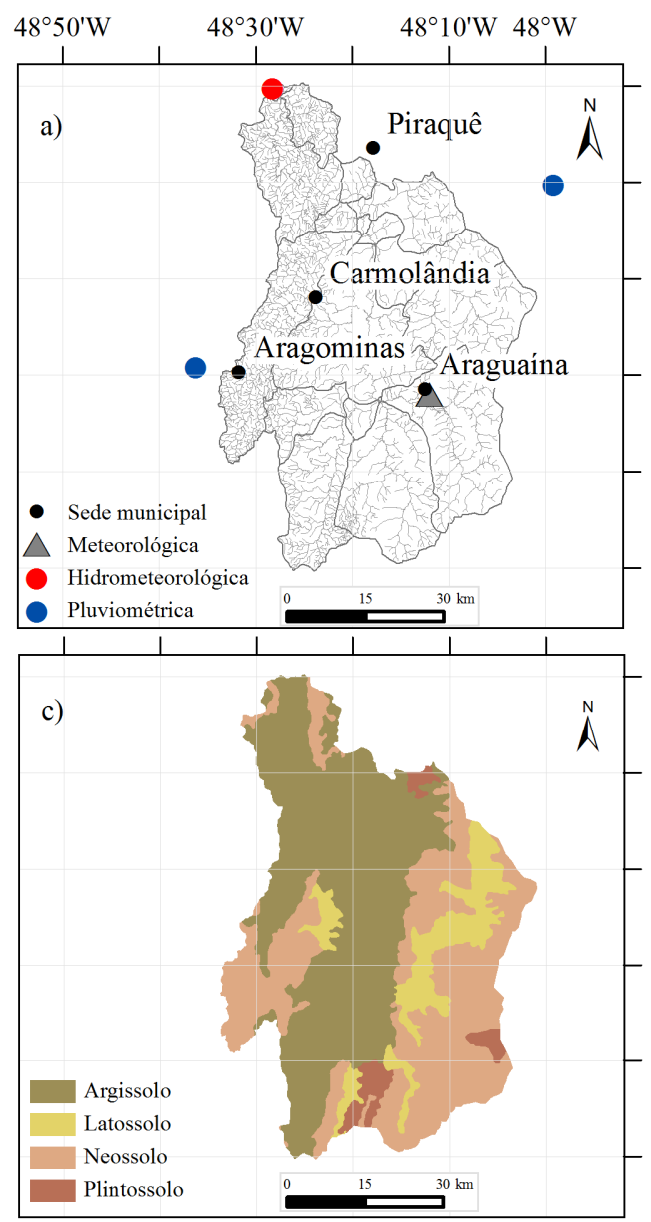

suprir a demanda populacional (54\%) e a industrial (7\%) (Magna, 2002). De acordo com a compartimentação geoambiental do Zoneamento Agroecológico do Tocantins, a região de cabeceira e média bacia do rio Lontra inserem-se predominantemente nas Depressões e Patamares de Ananás e Araguaína, ao passo que a baixa bacia insere-se nas Serras e Serranias de Xambioá e na Depressão de Xambioá (SEPLAN, 2012). A altitude varia entre 151 e 533 metros, com relevo caracterizado por declividades inferiores a $10 \%$ a leste, enquanto que a oeste, os declives podem superar 45\% (SEPLAN, 2012). Os solos predominantes são os Argissolos (48,5\%) e Neossolos (37,5\%), acompanhados de manchas de Plintossolos (3,2\%) e Latossolos (10,8\%) (SEPLAN, 2012). Na Figura 3 estão apresentados os mapas base para simulação hidrológica da bacia hidrográfica do rio Lontra, a partir dos quais foram estabelecidos os parâmetros fixos que caracterizam as subunidades modeladas, conforme descrevem Mello et al. (2008), Viola et al. (2009) e Beskow et al. (2011).

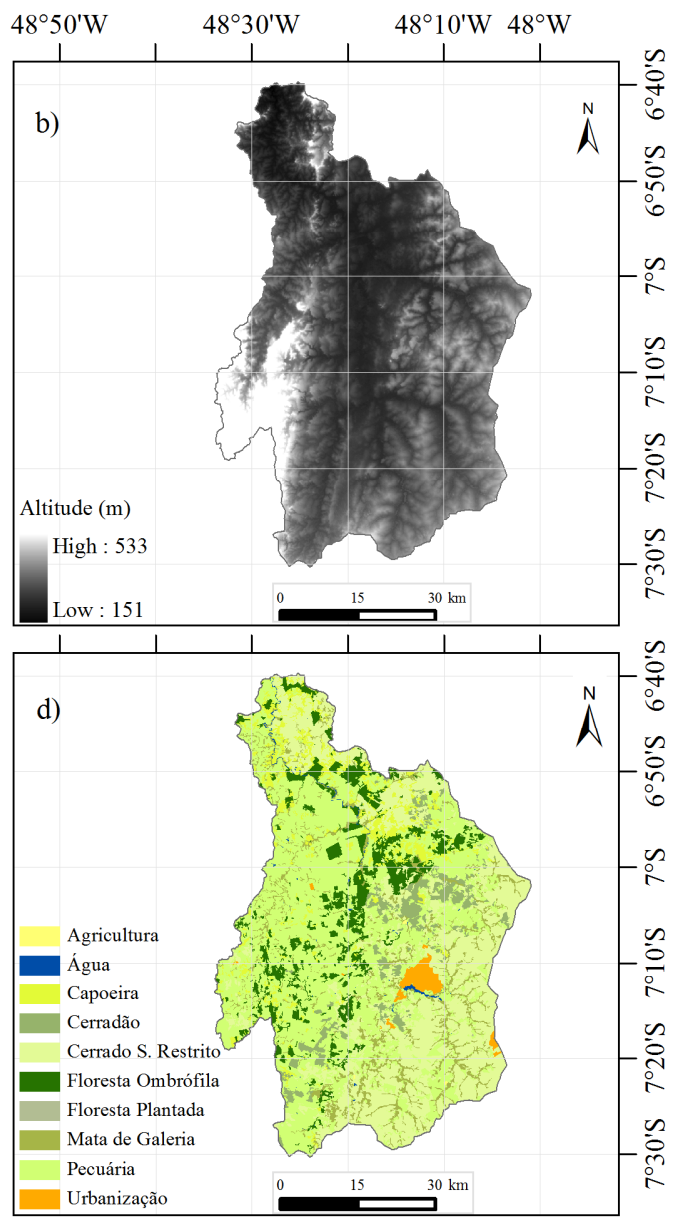

Figura 3 - Postos de monitoramento hidro-climatológico e sub-bacias (a), modelo digital de elevação (b), mapa de solos (c) (SEPLAN, 2012) e mapa de uso do solo (d) (Adaptado de SEPLAN, 2012). 
O modelo LASH é caracterizado como determinístico, semiconceitual e distribuído por sub-bacias (Viola, 2008; Beskow, 2009; Beskow et al., 2011). A modelagem é dividida em três módulos básicos da seguinte forma: (a) no primeiro, estimam-se as lâminas de escoamento superficial direto $\left(\mathrm{D}_{\mathrm{SUP}}\right)$, subsuperficial $\left(\mathrm{D}_{\mathrm{SS}}\right)$ e subterrâneo $\left(\mathrm{D}_{\mathrm{B}}\right)$; (b) o segundo simula o retardamento dos escoamentos nas sub-bacias através de reservatórios lineares e estimativas de tempos de residência da água em cada módulo; (c) as vazões são propagadas na rede de drenagem através de um modelo vazão-vazão que, no caso, foi aplicado o modelo Muskingan-Cunge Linear.

A estimativa dos processos físicos do ciclo hidrológico envolvidos na modelagem é baseada no armazenamento atual de água no solo. A cada passo do modelo essa variável é atualizada no balanço hídrico, de acordo com a Equação 1. Detalhes adicionais sobre este modelo podem ser obtidos nos trabalhos desenvolvidos por Mello et al. (2008), Viola et al. (2009), Beskow et al. (2011).

$$
\mathrm{A}_{\mathrm{t}+1}=\mathrm{A}_{\mathrm{t}}+\left(\mathrm{P}-\mathrm{D}_{\mathrm{SUP}}-\mathrm{D}_{\mathrm{SS}}-\mathrm{D}_{\mathrm{B}}-\mathrm{ET}\right) \cdot \Delta \mathrm{t}
$$

em que $A_{t+1}$ é o armazenamento de água no solo no tempo $t+1 ; A_{t}$ é o armazenamento no tempo anterior; $\mathrm{P}$ é a precipitação descontada a interceptação; $D_{\text {SuP }}$ é a lâmina de escoamento superficial; $D_{S S}$ é a lâmina de escoamento subsuperficial; $D_{B}$ é a lâmina de escoamento subterrâneo; ET é a evapotranspiração e $\Delta \mathrm{t}$ é o intervalo ou passo de simulação. Todos os componentes estão em mm dia ${ }^{-1}$.

A simulação hidrológica da bacia hidrográfica do rio Lontra foi desenvolvida entre os anos de 1995 e 2008, sendo aplicado o período de 1995 a 2001 para a calibração do modelo hidrológico e o de 2002 a 2008 para a sua validação. Para o ajuste dos parâmetros calibráveis foi aplicado o método de calibração automático, respeitando a faixa de valores associada a cada parâmetro, conforme descrito por Mello et al. (2008). Como, no início da simulação, ocorrem incertezas associadas ao desconhecimento das condições hidrológicas iniciais, especialmente a umidade inicial do solo, justifica-se a introdução de um período para aquecimento (warm up) (Stackelberg et al., 2007; Zhang et al., 2007). Nesse sentido, os anos de 1995 e de 2002 foram reservados para o aquecimento nas etapas de calibração e validação, respectivamente.
Para avaliar a eficácia da simulação foram aplicados os seguintes coeficientes estatísticos: coeficiente de Nash-Sutcliffe $\left(\mathrm{C}_{\mathrm{NS}}\right)$ e sua versão logarítmica $\left(\log \left(\mathrm{C}_{\mathrm{NS}}\right)\right)$ (Nash e Sutcliffe, 1970); coeficiente de determinação $\left(R^{2}\right)$ (Legates e McCabe, 1999); e desvio entre vazões observadas e simuladas (D) (Liew et al., 2003). Na sequência, estão apresentadas suas formulações, em que $\mathrm{Q}_{\mathrm{oBs}}$

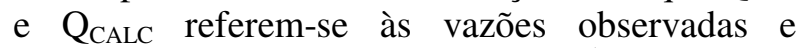
calculadas, respectivamente, $\mathrm{em} \mathrm{m}^{3} \mathrm{~s}^{-1}$.

De acordo com Zhang et al. (2007), o valor do coeficiente Nash-Sutcliffe indica o ajustamento dos dados simulados aos observados na reta $1: 1$, podendo variar de $-\infty$ a $1 . \mathrm{O}_{\mathrm{NS}}$ está associado à eficiência de estimativa de vazões de pico do hidrograma. Moriasi et al. (2007) apresentaram a seguinte classificação para este coeficiente, utilizando passo diário de simulação: $\mathrm{C}_{\mathrm{NS}}>0,65 \mathrm{o}$ modelo é considerado muito bom; $0,54<\mathrm{C}_{\mathrm{NS}}<$ 0,65 o modelo é considerado bom e entre 0,5 e 0,54, satisfatório. Segundo Zaapa (2002), valores de $\mathrm{C}_{\mathrm{NS}}$ acima de 0,5 qualificam o modelo para simulação.

$$
\begin{gathered}
\mathrm{C}_{\mathrm{NS}}=1-\frac{\sum_{\mathrm{i}=1}^{\mathrm{N}}\left(\mathrm{Q}_{\mathrm{OBS} \mathrm{i}}-\mathrm{Q}_{\mathrm{CALCi}}\right)^{2}}{\sum_{\mathrm{i}=1}^{\mathrm{N}}\left(\mathrm{Q}_{\mathrm{OBS} \mathrm{i}}-\overline{\mathrm{Q}_{\mathrm{OBS}}}\right)^{2}} \\
\log \left(\mathrm{C}_{\mathrm{NS}}\right)=1-\frac{\sum_{\mathrm{i}=1}^{\mathrm{N}}\left(\log \left(\mathrm{Q}_{\mathrm{OBSi}}\right)-\log \left(\mathrm{Q}_{\mathrm{CALCi}_{\mathrm{i}}}\right)\right)^{2}}{\sum_{\mathrm{i}=1}^{\mathrm{N}}\left(\log \left(\mathrm{Q}_{\mathrm{OBSi}}\right)-\overline{\log \left(\mathrm{Q}_{\mathrm{OBSi}}\right)}\right)^{2}}
\end{gathered}
$$

$\mathrm{O}$ valor de $\mathrm{R}^{2}$, de acordo com Legates e McCabe (1999), é um indicador da correlação entre os valores observados e simulados, com amplitude de variação entre 0 e 1, em que o valor 1 indica um ajuste perfeito. Esse coeficiente é considerado uma das estatísticas mais sensíveis a valores extremos (Legates e Mccabe, 1999). Moriasi et al. (2007) consideram valores de $\mathrm{R}^{2}$ acima de 0,5 como aceitáveis.

$$
\mathrm{R}^{2}=\left\{\frac{\sum_{\mathrm{i}=1}^{\mathrm{N}}\left(\mathrm{Q}_{\mathrm{OBSi}}-\overline{\mathrm{Q}_{\mathrm{OBB}}}\right) \cdot\left(\mathrm{Q}_{\mathrm{CALG}}-\overline{\mathrm{Q}_{\mathrm{CALC}}}\right)}{\left[\sum_{\mathrm{i}=1}^{\mathrm{N}}\left(\mathrm{Q}_{\mathrm{OBSi}}-\overline{\mathrm{Q}_{\mathrm{OBS}}}\right)^{2}\right]^{0,5} \cdot\left[\sum_{\mathrm{i}=1}^{\mathrm{N}}\left(\mathrm{Q}_{\mathrm{CALG}}-\overline{\mathrm{Q}_{\mathrm{CALC}}}\right)^{2}\right]^{0,5}}\right\}
$$

$\mathrm{O}$ valor de $\mathrm{D}$ significa a tendência média das estimativas produzidas pelo modelo e, quando positivo, expressa tendência de superestimativa e, 
quando negativo, de subestimativa. Liew et al. (2003) apresentam os seguintes intervalos e respectivas interpretações de $\mathrm{D}:<10 \%$, muito bom; entre $10 \%$ e $15 \%$, bom; entre $15 \%$ e $25 \%$, satisfatório e $>25 \%$, o modelo produz estimativas inadequadas no tocante à tendência.

$$
\mathrm{D}(\%)=\frac{\sum_{\mathrm{i}=1}^{\mathrm{N}}\left(\frac{\mathrm{Q}_{\text {estimado(i) }}-\mathrm{Q}_{\text {obs(i) }}}{\mathrm{Q}_{\mathrm{obs}(\mathrm{i})}}\right) \cdot 100}{\mathrm{~N}}
$$

\section{RESULTADOS E DISCUSSÃO}

$\mathrm{Na}$ Figura 4 estão apresentados os coeficientes estatísticos, hietograma e hidrogramas observado e simulado para a bacia hidrográfica do rio Lontra nas etapas de calibração e validação.

Os coeficientes estatísticos mostraram bom desempenho do modelo LASH na simulação contínua de vazões com passo diário na bacia hidrográfica do rio Lontra. $\mathrm{O}$ coeficiente $\mathrm{C}_{\mathrm{NS}}$ atingiu o patamar superior das classificações propostas por Zappa (2002) e Moriasi et al. (2007). Este resultado indica boa performance no ajuste das vazões de pico, que é uma das etapas mais complexas de um processo de simulação hidrológica.
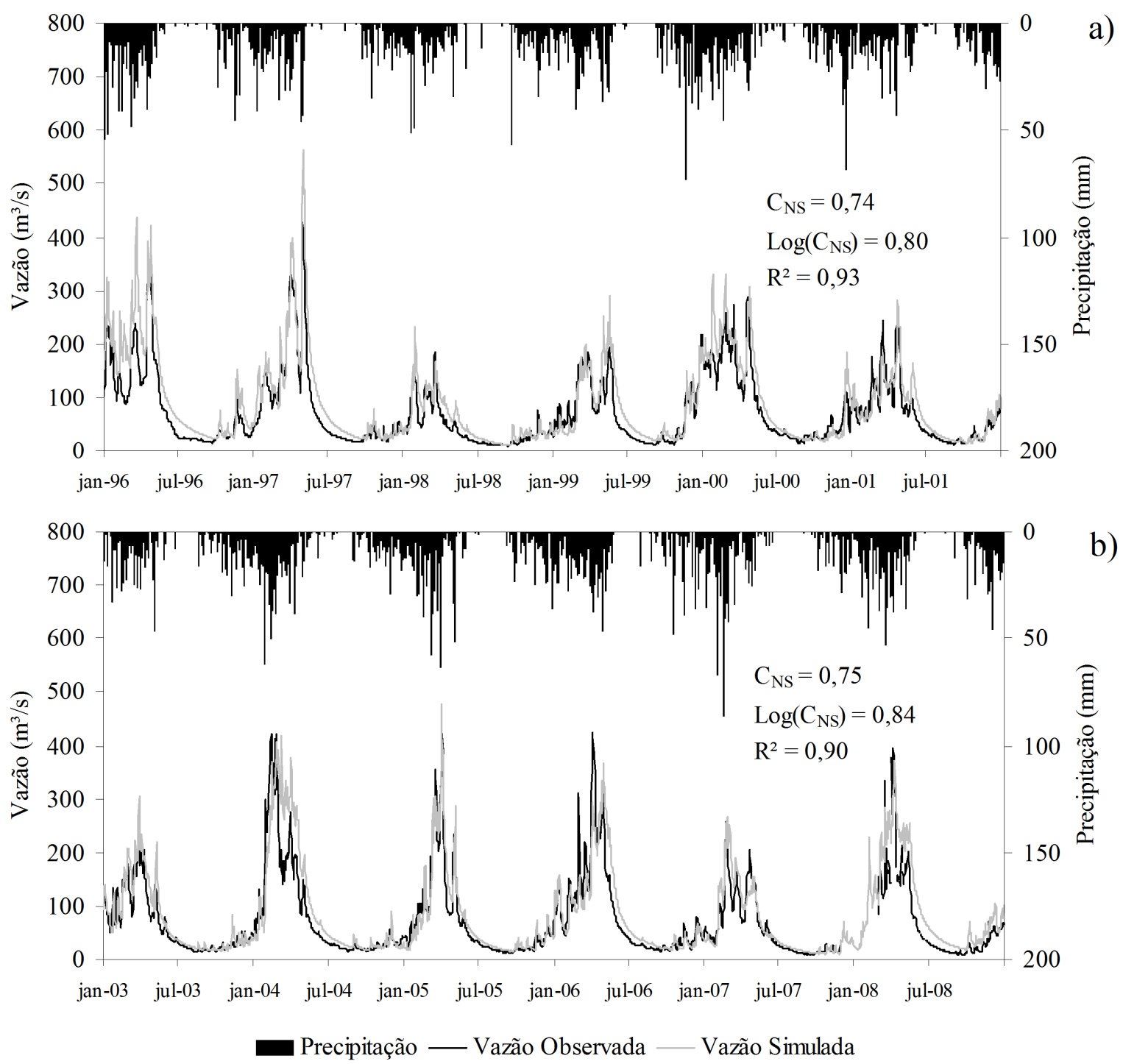

Figura 4 - Hidrograma observado, hidrograma simulado, hietograma observado e coeficientes estatísticos para a bacia hidrográfica do rio Lontra, durante a calibração (a) e verificação (b). 
Estudos recentes sobre a aplicação de modelos hidrológicos em diferentes realidades físicas utilizaram esse coeficiente para avaliar a acurácia da simulação. Notter et al. (2007), na região do Monte Kenya, África, desenvolveram e aplicaram um modelo hidrológico baseado no método $\mathrm{CN}$ SCS, obtendo $C_{N S}$ da ordem de 0,69 na calibração e de 0,51 na verificação. Licciardelo et al. (2007) aplicaram o modelo hidrológico AGNPS a uma bacia hidrográfica da região do Mediterrâneo, no sul da Itália e obtiveram $\mathrm{C}_{\mathrm{NS}}$ de 0,77 e 0,80 na calibração e verificação, respectivamente. Collischonn et al. (2005) e Thanapakpawin et al. (2007) aplicaram modelos hidrológicos distribuídos em bacias da Tailândia e Brasil, respectivamente, obtendo $\mathrm{C}_{\mathrm{NS}}$ da ordem de 0,75. Mello et al. (2008) e Viola et al. (2009) aplicaram o modelo LASH na bacia hidrográfica do rio Aiuruoca, obtendo $\mathrm{C}_{\mathrm{NS}}$ de 0,87 e 0,92 na calibração e verificação, respectivamente. Mello et al. (2008) avaliaram a aplicabilidade do modelo LASH para simular os impactos hidrológicos devido a alterações no uso do solo na bacia do rio Grande. Os autores avaliaram o efeito de diferentes períodos de tempo para calibração e validação, obtendo $C_{N S}$ superior a 0,75 em todas as abordagens. Beskow (2009) desenvolveu uma estrutura distribuída para o modelo LASH e a aplicou na simulação de uma pequena bacia da região Alto Rio Grande, com $\mathrm{C}_{\mathrm{NS}}$ de 0,82 para a calibração e de 0,76 para a verificação.

A avaliação do escoamento durante o período de vazante foi realizada pelo coeficiente estatístico $\log \left(\mathrm{C}_{\mathrm{NS}}\right)$. Resultados deste coeficiente atestaram alta confiabilidade na simulação dos períodos de recessão do hidrograma de acordo com as classificações de Moriasi et al. (2007) e Zappa (2002). Aliado a isto, os bons resultados do coeficiente de determinação $\left(\mathrm{R}^{2}\right)$ atestaram a viabilidade do modelo como ferramenta para a gestão de recursos hídricos, em bacias hidrográficas em região de tensão ecológica, como é o caso da bacia do rio Lontra, no norte do Tocantins. Estudos hidrológicos desenvolvidos com as mesmas finalidades e que apresentaram coeficientes estatísticos da mesma qualidade foram considerados precisos e aptos para a simulação, como os de Bormann et al. (2007), para uma bacia hidrográfica na Alemanha, Marsik e Waylen (2006) na Costa Rica, Licciardello et al. (2007) na Itália, Notter et al. (2007) no Quênia, Collischonn et al. (2005) no Rio Grande do Sul, Thanapakpawin et al. (2007) na Tailândia; Beskow et al. (2011) e Mello et al. (2008) na região Alto Rio Grande, sul de Minas Gerais.

Pode-se observar, nas etapas de calibração e validação do modelo hidrológico LASH na bacia hidrográfica do rio Lontra, que a tendência média das estimativas foram positivas, sendo de $24,4 \%$ e $21 \%$, respectivamente. Segundo a classificação proposta por van Liew et al. (2003), associada ao comportamento médio dos desvios, os resultados são classificados como satisfatórios. Em modelos hidrológicos, de complexa formulação e com grande número de parâmetros, estes valores de tendência podem ser considerados baixos, significando que o modelo produzirá estimativas, além de precisas, não enviesadas, o que consiste de uma característica indispensável de qualquer modelo matemático.

As curvas de permanência observada e simulada e a dispersão de seus principais valores de referência em torno da reta 1:1 estão apresentadas na Figura 5. Essa abordagem evidencia a frequência com a qual determinada vazão é excedida e permite avaliar a acurácia do modelo na simulação de vazões mínimas e máximas.
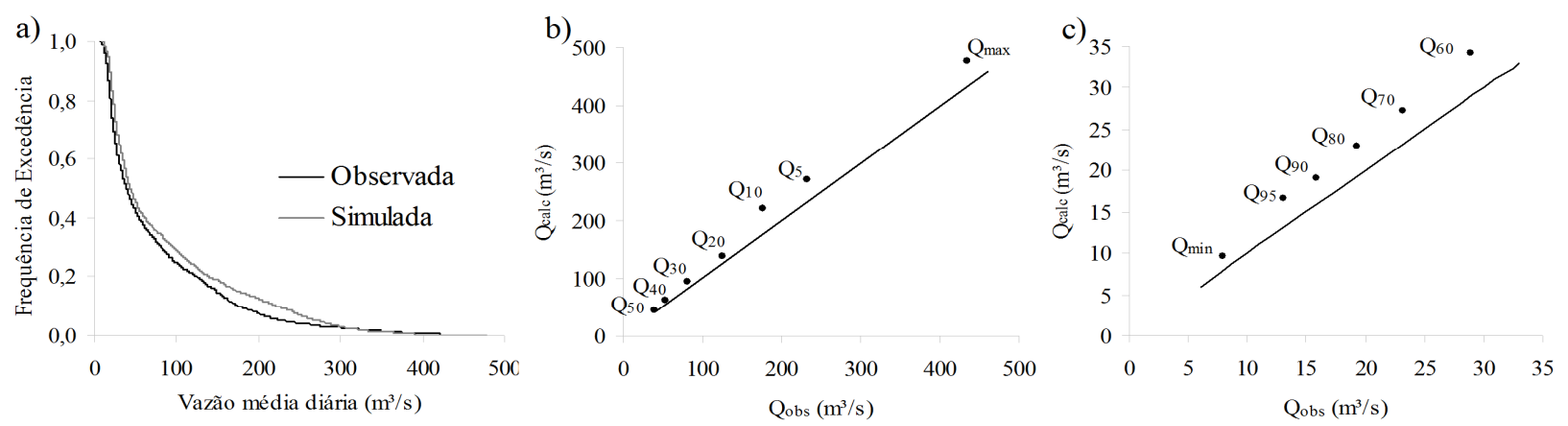

Figura 5 - Curva de permanência observada e simulada (a), e a dispersão de seus principais valores de referência máximos (b) e mínimos (c) em torno da reta 1:1. 
Os impactos negativos provocados por grandes cheias podem ser minimizados pela previsão de vazões, que se fundamenta no acoplamento de um modelo hidrológico a um sistema de previsão do tempo ou a uma rede de observação meteorológica com transmissão dos dados em tempo real. Conforme se pode observar, a curva de permanência simulada mostrou forte aderência à observada. No que tange aos valores de menor frequência (maiores vazões), visualiza-se ótimo ajuste das vazões com permanência de $50 \%\left(\mathrm{Q}_{50}\right)$, $40 \%\left(\mathrm{Q}_{40}\right), 30 \%\left(\mathrm{Q}_{30}\right)$ e $20 \%\left(\mathrm{Q}_{20}\right)$. Pequenas superestimativas, de $24,8 \%, 16,7 \%$ e $9,5 \%$ foram encontradas para $\mathrm{Q}_{10}, \mathrm{Q}_{5}$ e para a vazão máxima $\left(\mathrm{Q}_{\max }\right)$ do período analisado (2003 a 2008), respectivamente. Estes resultados estão em consonância com aqueles associados às estatísticas de precisão e atestam a performance do modelo para estimativa de vazões máximas, demonstrando as qualidades necessárias para a previsão de vazões para uso pela vigilância civil, altamente relevante para minimizar os danos decorrentes de grandes cheias, bem como para o desenvolvimento de estudos associados aos períodos de ascensão do hidrograma. Notter et al. (2007), Stackelberg et al. (2007) e Viola et al. (2009), em estudo desenvolvido com a mesma finalidade obtiveram resultados semelhantes e destacaram que a principal limitação para a modelagem das vazões máximas está associada à dificuldade de representar a distribuição espacial e temporal das chuvas, e também ao passo da modelagem, que muitas vezes pode ser superior ao tempo de concentração das sub-bacias.

A principal funcionalidade da curva de permanência é na determinação de vazões mínimas de referência para outorga, no contexto da gestão de recursos hídricos. No estado do Tocantins, o decreto $\mathrm{n}^{\circ} 2.432$ de junho de 2005, estabelece a vazão de referência para outorga como sendo a com $90 \%$ de permanência $\left(\mathrm{Q}_{90}\right)$. O valor de $\mathrm{Q}_{90}$ observado para o período em análise foi de $15,8 \mathrm{~m}^{3} \mathrm{~s}^{-1}$, enquanto que o estimado foi de $19 \mathrm{~m}^{3} \mathrm{~s}^{-1}$, o que representa um erro de $20,2 \%$. Para as demais vazões em análise os erros foram de $18,4 \%, 18 \%, 19,3 \%$ e $26,6 \%$, respectivamente para $\mathrm{Q}_{60}, \mathrm{Q}_{70}, \mathrm{Q}_{80}$ e $\mathrm{Q}_{95}$, com importante resultado para a vazão mínima, visto que o erro de estimativa desta foi de $22,4 \%$. Mello et al. (2008) calibrou o modelo hidrológico LASH para a região de cabeceira do rio Grande, no sul de Minas Gerais, tendo encontrado um erro de $25,2 \%$ para $\mathrm{Q}_{90}$. Ainda neste sentido, Beskow (2009) calibrou o modelo LASH para a bacia hidrográfica do ribeirão Jaguara, e obteve uma estimativa de 0,131 $\mathrm{m}^{3} \mathrm{~s}^{-1}$ para $\mathrm{Q}_{90}$, comparado a um valor observado de $0,122 \mathrm{~m}^{3} \mathrm{~s}^{-1}$. De acordo com os autores esta magnitude de desvio atesta a aplicabilidade do modelo hidrológico, uma vez que os erros obtidos por modelos empíricos de regionalização hidrológica são frequentemente muito superiores. Desta maneira, os resultados do período de validação do modelo hidrológico na bacia hidrográfica do rio Lontra, forneceram uma curva de permanência de boa qualidade, permitindo estimativas confiáveis de vazões de referência para outorga, indicando potencial para aplicação na gestão de recursos hídricos na região norte do Tocantins.

\section{CONCLUSÕES}

Os resultados dos coeficientes estatísticos aplicados para avaliar o desempenho do modelo hidrológico LASH mostraram boa performance nas etapas de calibração e validação, atestando a qualidade do modelo para demandas associadas à gestão e planejamento dos recursos hídricos na sub-bacia hidrográfica do rio Lontra.

As curvas de permanência simuladas pelo modelo com passo diário mostraram forte aderência às observadas, com pequenos erros de estimativa, inclusive para a vazão mínima e para a vazão máxima do período analisado. Estes resultados evidenciam a aplicabilidade do modelo em distintas demandas enfrentadas pela gestão e planejamento dos recursos hídricos no estado do Tocantins.

\section{REFERÊNCIAS}

Aquino, S.; Latrubesse, E. M.; Souza Filho, E. E. de. (2009), Caracterização hidrológica e geomorfológica dos afluentes da bacia do rio Araguaia. Revista Brasileira de Geomorfologia, 10, 43-54.

ANA. Agência Nacional de Águas. Bacia Hidrográfica dos Rios Tocantins-Araguaia. Disponível em <http:// http://www2.ana.gov.br/Paginas/servicos/planejam ento/planoderecursos/Tocantins-Araguaia $>$ Acesso em: 27 jul. 2012.

Beskow, S. LASH model: a hydrological simulation toll in gis framework. Tese (Doutorado 
em Engenharia Agrícola) - Universidade Federal de Lavras, 2009.

Beskow, S.; Mello, C. R.; Norton, L. D.; da Silva, A. M. (2011), Performance of a distributed semiconceptual hydrological model under tropical watershed conditions. Catena, 86, 160-171.

BDMEP: Banco de dados. Disponível em : < http://www.inmet.gov.br/projetos/rede/pesquisa/>. Acesso em: 10 mar. 2012.

Bormann, H.; Breuer, L.; Graff, T.; Huisman, J. A. (2007), Analyzing the effects of soil properties changes associated with land use changes on the simulated water balance: a comparison of three hydrological catchment models for scenario analysis. Ecological Modelling, 209, 29-40.

Collischonn, W.; Tucci, C.E.M.; Haas, R.; Andreolli, I. (2005), Forecasting River Uruguay flow using rainfall forecasts from a regional weather-prediction model. Journal of Hydrology, 305, 87-98.

Henry, R. Ecótonos nas interfaces dos ecossistemas aquáticos. Ed. Rima, São Carlos, 350p., 2003

HIDROWEB: banco de dados. Disponível em : < http://hidroweb.ana.gov.br/>. Acesso em: 10 mar. 2012.

IBGE, Instituto Brasileiro de Geografia e Estatística. 2004. Mapa de Biomas do Brasil. Disponível em: <www.ibge.gov.br>. Acesso em: 05 jul. 2010.

Kark, S. and Rensburg, B. J. (2006), Van. Ecotones: Marginal or central areas of transition? Israel Journal of Ecology and Evolution, 52, 2953, 2006.

Latrubesse, E. and Stevaux, J. C. (2002), Geomorphology and environmental aspects of Araguaia fluvial basin, Brazil. Geomorphologie, 129, 109-127.

Legates, D. R. and Mccabe, G. J. (1999), Evaluating the use of "goodness of fit" measures in hydrologic and hydroclimatic model validation. Water Resources Research, 26, 69-86.
Licciardelo, F.; Zema, D. A.; Zimbone, S. M.; Bingner, R. L. (2007), Runoff and soil erosion evaluation by the AnnAGNPS model in a small Mediterranean Watershed. Transactions of the $A S A B E$, 50, 1585-1593.

Liew, M. W. Van; Arnold, J. G.; Garbrecht, J. D. (2003), Hydrologic simulation on agricultural watersheds: choosing between two models. Transactions of the ASAE, 46, 1539-1551.

MAGNA ENGENHARIA LTDA. Plano de recursos hídricos das bacias hidrográficas dos rios Lontra e Corda, na região do bico do Papagaio/TO. Relatório: Palmas, 2002.

Malanson, G. P. (997), Effects of feedbacks and seed rain on ecotone patterns. Landscape Ecology, $12,27-38$.

Marsik, M. and Waylen, P. (2006), An application of the distributed hydrologic model CASC2D to a tropical montane watershed. Journal of Hydrology, 330, 481-495.

Mello, C. R.; Viola, M. R.; Norton, L. D.; Silva, A. M.; Weimar, F. A. (2008), Development and application of a simple hydrologic model simulation for a Brazilian headwater basin. Catena, 75, 235-247.

Moriasi, D. N.; Arnold, J. G.; Liew, M. W. Van; Binger, R. L.; Harmel, R. D.; Veith, T. (2007), Model evaluation guidelines for systematic quantification of accuracy in watershed simulations. Transactions of the ASABE, 50, 885900.

Nash, J. E. and Sutcliffe, J. V. (1970), River flow forecasting through conceptual models part I: a discussion of principles. Journal of Hydrology, 10, 282-290.

Nobrega, M. T.; Collischonn, W.; Tucci, C. E. M.; Paz, A. R. (2011), Uncertainty in climate change impacts on water resources in the Rio Grande Basin, Brazil. Hydrology and Earth System Sciences, 15, 585-595.

Notter, B.; Macmillan, L.; Viriroli, D.; Weingartner, R.; Liniger, H. P. (2007), Impacts of environmental change on water resources in the 
Mt. Kenya Region. Journal of Hydrology, 343, 266-278.

Ribeiro Neto, A.; Silva, R. C. V.; Collischonn, W.; Tucci, C. E. (2008), Simulação na Bacia Amazônica com Dados Limitados: Rio Madeira. Revista Brasileira de Recursos Hídricos, 13, 47-58.

SECRETARIA DO PLANEJAMENTO E DA MODERNIZAÇÃO DA GESTÃO PÚBLICA. Mapeamento das Regiões Fitoecológicas e Inventário Florestal do Estado do Tocantins. Palmas: SEPLAN, 2011.

SECRETARIA DO PLANEJAMENTO E DA MODERNIZAÇÃO DA GESTÃO PÚBLICA. Atlas do Tocantins: subsídios ao planejamento da gestão territorial. 6. Ed. rev. atu. Palmas: SEPLAN, 2012.

SECRETARIA DO MEIO AMBIENTE E DESENVOLVIMENTO SUSTENTÁVEL. Plano Estadual de Recursos Hídricos. Palmas: SEMADES, 2011.

Stackelberg, N. O. Von; Chescheir, G. M.; Skaggs, R. W.; Amatya, D. M. (2007), Simulation of the hydrologic effects of afforestation in the Tacuarembó River Basin, Uruguay. Transactions of the ASABE, 50, 455-468.

Thanapakpawin, P.; Richey, J.; Thomas, D.; Rodda, S.; Campbell, B.; Logsdon, M. (2007), Effects of landuse change on the hydrologic regime of the Mae Chaem river basin, NW Thailand. Journal of Hydrology, 334, 215-230.
Viola, M. R. Simulação hidrológica na Região Alto Rio Grande a montante do reservatório de Camargos/CEMIG. Dissertação (Mestrado em Engenharia Agrícola) - Universidade Federal de Lavras, 2008.

Viola, M. R.; Mello, C. R.; Acerbi Junior, F. W.; Silva, A. M. (2009), Modelagem hidrológica na bacia hidrográfica do Rio Aiuruoca, MG. Revista Brasileira de Engenharia Agrícola e Ambiental, 13, 581-590.

Viola, M. R. Simulação hidrológica na cabeceira da bacia hidrográfica do rio Grande de cenários de usos do solo e mudanças climáticas A1B. Tese (Doutorado em Recursos Hídricos em Sistemas Agrícolas) - Universidade Federal de Lavras, 2011.

Zaapa, M. Multiple-response verification of a distributed hydrological model at different spatial scales. Thesis (Ph.D. in Natural Science) - Swiss Federal Institute of Technology, 2002.

Zhang, H. G.; Fu, S. H.; Fang, W. H.; Imura, H.; Zhang, X. C. (2007), Potential effects of climate change on runoff in the Yellow River Basin of China. Transactions of the ASABE, 50, 911-918. 